\title{
Pancytopenia secondary to hypothyroidism in a 13-year- old male child
}

\author{
Gkampeta $A^{1 *}$, Stamou $M^{1}$, Kotsa $K^{2}$, Lazaridou $N^{1}$, Hatzipantelis $E^{1}$, Pantoleon $A^{3}$ and Giannopoulos $A^{1}$ \\ ${ }^{1} 2^{\text {nd }}$ Pediatric Department, Aristotle University of Thessaloniki, AHEPA General Hospital, Greece \\ ${ }^{2}$ Division of Endocrinology and Metabolism, 1st Department of Internal Medicine, AHEPA University Hospital, Aristotle University of Thessaloniki, Greece \\ ${ }^{3}$ Department of Radiology, Aristotle University of Thessaloniki, "AHEPA” Hospital, Greece
}

\begin{abstract}
Thyroid hormones have a crucial role in erythropoiesis and metabolism. They enhance erythropoiesis through hyper proliferation of immature erythroid progenitors and increase secretion of erythropoietin by inducing erythropoietin gene expression. Thyroid dysfunction has various effects on blood cells such as anaemia, erythrocytosis leukopenia, thrombocytopenia, and in rare cases pancytopenia. The exact pathogenesis of anaemia secondary to hypothyroidism is not precisely explained but appears to reflect an adaptation to decreased tissue oxygen requirements resulting from a decrease in the basal metabolic rate. In reviewing the literature, there have been few previously reported cases of hematological abnormalities due to hypothyroidism, all of them concerning adults. In this article, we describe a rare case of pancytopenia secondary to hypothyroidism in a 13-year-old male child and its partial reversal after thyroid hormone replacement. Hypothyroidism should be considered in patients with unexplained pancytopenia. Further studies are needed to determine the exact underlying disease mechanism.
\end{abstract}

\section{Introduction}

The thyroid gland synthesizes and secrets two major hormones, known as 3, 5, 3'-triiodothyronine (T3) and 3, 5, 3', 5'-tetraiodothyronine (T4) or thyroxin, which have critical role in early brain development, somatic growth, bone maturation, protein synthesis and regulate production of red blood cells. Thyroid hormones also involve in hemoglobin production and maturation of hemoglobin in fetus [1].

In hypothyroidism anaemia of a mild degree is commonly present. Characteristically, the anaemia of hypothyroidism is normochromic and normocytic and is not correlated with the severity of the hypothyroidism. Less commonly it can be macrocytic or microcytic. Hypothyroidism also causes thrombocytopenia, leukopenia and in rare cases pancytopenia [2].

In this article, we describe a rare case of pancytopenia secondary to hypothyroidism in a 13-year-old male child.

\section{Case report}

A 13-year-old male child admitted to our department with a 24 hours history of febrile illness, headache and fatigue. One hour before the arrival he experienced an episode of loss of consciousness lasting 15 seconds. He had no significant past medical history and an unremarkable perinatal history (he was born full term, weighting 3.500 gr, with Apgar score $1^{10}$ and $\left.5^{10}\right)$. He was not taking any medication and there was a family history of Hashimoto's thyroiditis.

On physical examination he was in good general condition with a temperature of $36.4{ }^{\circ} \mathrm{C}$, blood pressure $95 / 53 \mathrm{~mm} \mathrm{Hg}$, heart rate 92 beats per minute, and $\mathrm{Spo}_{2} 99 \%$. On examination, he appeared with dry skin, alopecia areata, myxedema, typical hypothyroid facial features and delayed relaxation of deep tendon reflexes. The rest clinical examination was unremarkable.
Laboratory analysis revealed the following: WBC: $3 \times 10^{3} / \mu \mathrm{L}$

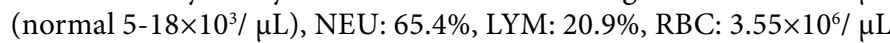
(normal 2.8-5.3×106/ $\mu \mathrm{L}$ ), HGB: $11.5 \mathrm{~g} / \mathrm{dl}$ (normal 10-17 g/dl), HCT: 34.4\% (normal 37-52\%), MCV: 96.9 fl, $\mathrm{MCH} 32.4$ pg, PLT: $98 \times 10^{3} /$ $\mu \mathrm{L}$ (normal $150-400 \times 10^{3} / \mu \mathrm{L}$ ), ferritin: $318.7 \mathrm{ng} / \mathrm{ml}$, Erythrocyte Sedimentation Rate: 37 mm/1 h, SGOT: 144 U/L (normal<80 U/L), SGPT: $184 \mathrm{U} / \mathrm{L}$ (normal<52 U/L), $\mathrm{K}^{+}: 4.3 \mathrm{mEq} / \mathrm{L}, \mathrm{Na}^{+}: 136 \mathrm{mEq} / \mathrm{L}$, $\mathrm{Ca}^{+2}$ : $9.19 \mathrm{mg} / \mathrm{dl}$, P: $3.6 \mathrm{mg} / \mathrm{dl}$, urea: $33 \mathrm{mg} / \mathrm{dl}$, creatinine: $1.89 \mathrm{mg} /$ dl, glucose: 83 g/dl, IgA: 93 mg/dl, IgG: $895 \mathrm{mg} / \mathrm{dl}, \operatorname{IgM}: 96 \mathrm{mg} / \mathrm{dl}$, IgE: $79.8 \mathrm{IU} / \mathrm{L}, \mathrm{C} 3: 74.3 \mathrm{mg} / \mathrm{dl}, \mathrm{C} 4: 15.3 \mathrm{mg} / \mathrm{dl}$, CRP: $1.130 \mathrm{mg} / \mathrm{dl}$, PT: $14.8 \mathrm{sec}$, APTT: $38 \mathrm{sec}$, INR: 1.22, Lactate Dehydrogenase: 450 U/L, creatine phosphokinase: $3690 \mathrm{U} / \mathrm{L}$, Vitamin B12: $441 \mathrm{pmol} / \mathrm{L}$, cortisol: $245 \mathrm{nmol} / \mathrm{L}$.

To exclude any neoplastic disease, we performed a bone marrow biopsy which revealed a hypocellular bone marrow and was not indicative of any hematological or neoplastic disease.

Further investigations revealed normal autoimmune profile (ANA, dsDNA, ANCA, Ro/SS - A, Ro/SS - B, Sm, RNP, Scl 70, Jo1). The patient's thyroid function was abnormal with thyroid stimulating hormone (TSH) $100 \mu \mathrm{IU} / \mathrm{ml}$ (normal, $0.3-3.0 \mu \mathrm{IU} / \mathrm{ml}$ ) and free T4 of $0.3 \mathrm{pmol} / \mathrm{L}$ (normal, $10-25 \mathrm{pmol} / \mathrm{L}$ ). Antithyroid peroxidase antibodies (Anti-TPO: $5 \mathrm{IU} / \mathrm{ml}$, normal $<35 \mathrm{IU} / \mathrm{mL}$ ) and thyroglobulin antibodies (Anti-TG: $42 \mathrm{IU} / \mathrm{ml}$, normal<20 IU/mL) were detectable. In evaluation of the thyroid gland, ultrasonography demonstrated a diffusely enlarged thyroid gland with a heterogeneous echotexture with the

${ }^{\star}$ Correspondence to: Anastasia Gkampeta, St. Kyriakidi 1, Thessaloniki, E-mail: anastagab@yahoo.gr

Key words: children, hypothyroidism, pancytopenia

Received: July 18, 2019; Accepted: August 16, 2019; Published: August 19, 2019 
presence of hypoechoic micronodules. Colour Doppler study showed normal flow. The radiologic appearance was most consistent with Hashimoto thyroiditis. The patient had normal response to the ACTH test (Synacthen test).

A diagnosis of hypothyroidism was made based on the thyroid hormone production levels and thyroid gland ultrasonography. The patient received intravenously fluid resuscitation and treatment with levothyroxine sodium, administered orally, was initiated. The patient's symptoms of fatigue, as well as his hematologic abnormalities, resolved rapidly.

On subsequent endocrine outpatient follow-up 3 months later, the patient's repeat blood counts (RBC, PLT) and also thyroid function hormones had normalized showing resolution of pancytopenia. However, WBC remain below normal levels (approximately $3-4 \times 10^{3} /$ $\mu \mathrm{L}$ ) within 3 months of thyroid hormone replacement. The informed consent was signed by patient's parents.

\section{Discussion}

Thyroid hormones have a crucial role in erythropoiesis and metabolism. They enhance erythropoiesis through hyper proliferation of immature erythroid progenitors and increase secretion of erythropoietin (EPO) by inducing EPO gene expression. Thyroid hormones also augment repletion of hypoxia inducible factor1 (HIF-1) and then motivate growth of erythroid colonies (BFU-E, CFU-E) [3].

Thyroid dysfunction has various effects on blood cells such as anaemia (normochromic-normocytic, hypochromic-microcytic or macrocytic), erythrocytosis leukopenia, thrombocytopenia, and in rare cases pancytopenia. Hypothyroidism can cause anaemia through reducing the oxygen metabolism. The exact pathogenesis of anaemia secondary to hypothyroidism is not precisely explained but appears to reflect an adaptation to decreased tissue oxygen requirements resulting from a decrease in the basal metabolic rate. Hypothyroid individuals with anaemia and normal serum iron, B12, and folate levels, have an increase in haemoglobin soon after thyroid hormone replacement [4].

Alteration in other hematological parameters such as hemoglobin (HG), hematocrit (HCT), mean corpuscular volume (MCV), mean corpuscular hemoglobin $(\mathrm{MCH})$, white blood cell count (WBC) and platelet count has also been associated with thyroid dysfunction. Pancytopenia is a rare side effect of hypothyroidism and its cause is not well understood. Immunological mechanisms have been associated with decline of erythrocytes' and platelets' life-span. Pancytopenia due to bone marrow hypoplasia has been reported in patients with myxoedema coma. Autoimmune reaction against bone marrow has been suggested as the underlying cause. Acton et al. [5] reported pancytopenia in a patient with hypopituitarism and autoimmune hypothyroidism which immediately resolved with initiation of corticosteroid and thyroid replacement therapy. The association between hypothyroidism and raised levels of hepatic transaminases, especially when muscle weakness is a symptom, in the blood has been described and possibly attributed to highly variable release of enzymes from cells resulting presumably from individual metabolic set-point [6]. These abnormalities of liver function were normalized in all published cases after hormone replacement therapy.

\section{Conclusion}

In reviewing the literature, there have been few previously reported cases of hematological abnormalities due to hypothyroidism, all of them concerning adults. There is one published report of an 11-year-old girl who developed hypothyroidism and prolonged pancytopenia after radiotherapy. The pancytopenia resolved after thyroxine replacement [7]. We describe a rare complication of hypothyroidism, pancytopenia, and its reversal after thyroid hormone replacement. Hypothyroidism should be considered in patients with unexplained pancytopenia. Further studies are needed to determine the exact underlying disease mechanism.

\section{Conflicts of interest}

The authors declared no potential conflicts of interest with respect to the research, authorship, and/or publication of this article. The authors received no financial support for the research, authorship, and/or publication of this article. The participation involved informed consent.

\section{References}

1. Shriraam M, Sridhar M (2014) Subclinical hypothyroidism in children. Indian Pediatr 51: $889-895$.

2. Tsoukas MA (2014) Pancytopenia in severe hypothyroidism. Am J Med 127: e11-12.

3. Dorgalaleh A, Mahmoodi M, Varmaghani B, Kiani Node F, Saeeidi Kia O, et al. (2013) Effect of thyroid dysfunctions on blood cell count and red blood cell indice. Iran J Ped Hematol Oncol 3: 73-77.

4. Rathi MS, Peacey SR (2011) Pancytopenia and nephrotic syndrome related to autoimmune hypothyroidism. JRSM Short Rep 2: 65.

5. Acton L, Chakraborty A, Thomson D, Wass J (2007) An unusual cause of pancytopaenia Acute Med 6: 77-78.

6. Saha B, Maity C (2002) Alteration of serum enzymes in primary hypothyroidism. Clin Chem Lab Med 40: 609-611.

7. Lee AC (2010) Pancytopenia secondary to hypopituitarism may just be due to hypothyroidism alone. Ann Hematol 89: 1181.

Copyright: (2019 Gkampeta A. This is an open-access article distributed under the terms of the Creative Commons Attribution License, which permits unrestricted use, distribution, and reproduction in any medium, provided the original author and source are credited. 